\title{
Using homological duality in consecutive pattern avoidance
}

\author{
Anton Khoroshkin * \\ Departement Matematik, ETH, \\ CH-8092, Zurich, Switzerland and \\ Lab. 170, ITEP, Moscow \\ RU-117259, Moscow, Russia \\ anton.khoroshkin@math.ethz.ch
}

\author{
Boris Shapiro \\ Department of Mathematics \\ Stockholm University \\ SE-106 91, Stockholm, SE \\ shapiro@math.su.se
}

Submitted: Sep 30, 2010; Accepted: May 16, 2011; Published: May 25, 2011

Mathematics Subject Classifications: 05A15, 05A05

\begin{abstract}
Using the approach suggested in [2] we present a sufficient condition guaranteeing that two collections of patterns of permutations have the same exponential generating functions for the number of permutations avoiding elements of these collections as consecutive patterns. In short, the coincidence of the latter generating functions is guaranteed by a length-preserving bijection of patterns in these collections which is identical on the overlappings of pairs of patterns where the overlappings are considered as unordered sets. Our proof is based on a direct algorithm for the computation of the inverse generating functions. As an application we present a large class of patterns where this algorithm is fast and, in particular, allows us to obtain a linear ordinary differential equation with polynomial coefficients satisfied by the inverse generating function.
\end{abstract}

\section{Introduction}

In recent years, the theory of consecutive pattern avoidance for permutations has experienced a rapid development since the publication of the important paper [5]. Among the latest publications one should mention [1], [9], [12], [4] where a number of special cases has been treated and the corresponding exponential generating functions explicitly found. The present text is devoted to the same topic and is an extension of the application of homological

*supported by RFBR 10-01-00836, RFBR-CNRS-10-01-93111, RFBR-CNRS-10-01-93113, and by the Federal Programm of Ministry of Education and Science of the Russian Federation under contract 14.740.11.0081 
methods to this theory (initiated in [2]). We investigate a natural analog of the notion of Wilf equivalence for consecutive pattern avoidance and obtain a rather general sufficient condition guaranteeing that this natural analog of Wilf equivalence holds. Most of the definitions below are borrowed from [2] and are rather standard in this area.

\subsection{Notation and definitions}

A permutation of length $n$ is a sequence $s=s(1), s(2), \ldots, s(n)$ containing each of the numbers $\{1, \ldots, n\}$ exactly once. To every sequence $s$ consisting of $n$ distinct positive integers, we associate its standardization st $[s]$, also known as the reduced form of $s$, which is the permutation of length $n$ uniquely determined by the condition that $s(i)<s(j)$ if and only if $\operatorname{st}[s](i)<\operatorname{st}[s](j)$. In other words, st $[s]$ is the unique permutation of length $n$ whose relative order of entries is the same as that of $s$. For example, st[573] $=231$. In what follows we will refer to distinct integers forming a permutation as its entries.

We say that a permutation $\sigma$ of length $n$ contains a permutation $\pi$ of length $l \leqslant n$ as a consecutive pattern if for some $i \leqslant n-l+1$ the standardization $\operatorname{st}[\sigma(i) \ldots \sigma(i+l-1)]$ coincides with $\pi$. If $\sigma$ contains $\pi$ as a consecutive pattern we say that $\pi$ divides $\sigma$ and use the notation $\pi \mid \sigma$. If $\pi \mid \sigma$ and $i=1$ (respectively $i=n-l+1$ ) we say that $\pi$ is a left (respectively right) divisor of $\sigma$. The main notion in the theory of pattern avoidance for permutations is as follows. We say that a permutation $\sigma$ avoids a given permutation $\pi$ as a consecutive pattern if $\sigma$ is not divisible by $\pi$. (Throughout this paper we only deal with consecutive patterns: the word "consecutive" will therefore be omitted.)

The central problem of the theory of pattern avoidance is to count the number of permutations of a given length avoiding a given collection $\Pi$ of forbidden patterns or, more generally, containing a given number of occurrences of patterns from $\Pi$. This problem naturally leads to the following equivalence relation on collections of patterns defined in the simplest case by $\mathrm{H}$. Wilf in [14]. Two collections of patterns $\Pi_{1}$ and $\Pi_{2}$ are said to be Wilf equivalent (denoted by $\Pi_{1} \simeq_{W} \Pi_{2}$ ) if for every positive integer $n$, the number of $\Pi_{1}$-avoiding permutations of length $n$ is equal to the number of $\Pi_{2}$-avoiding permutations of length $n$. We say that two collections of patterns $\Pi_{1}$ and $\Pi_{2}$ are strongly Wilf equivalent if for every positive integer $n$ and every nonnegative integer $0 \leqslant q \leqslant n$, the number of permutations of length $n$ with $q$ occurrences of patterns from $\Pi_{1}$ equals the number of permutations of length $n$ with $q$ occurrences of patterns from $\Pi_{2}$. In the set-up of consecutive pattern avoidance we will still speak about Wilf equivalent (respectively strongly Wilf equivalent) collections. (We use the notation: $\Pi_{1} \simeq_{W} \Pi_{2}$ for strongly Wilf equivalent collections.)

Remark 1.1. Throughout this paper we assume that every collection of patterns $\Pi$ is reduced, i.e., no two permutations $\pi, \pi^{\prime} \in \Pi$ are divisible by one another. Notice that if $\pi \mid \pi^{\prime} \in \Pi$ then $\Pi \backslash\left\{\pi^{\prime}\right\}$ is strongly Wilf equivalent to $\Pi$.

Following [5] consider two exponential generating functions in one and two variables respectively:

$$
\Pi(x):=\sum_{n} \alpha_{n} \frac{x^{n}}{n !} \quad \text { and } \quad \Pi(x, t):=\sum_{n, k} \alpha_{n, q} \frac{x^{n}}{n !} t^{q},
$$


associated to a given collection of patterns $\Pi$. Here $\alpha_{n}$ (respectively, $\alpha_{n, q}$ ) is the number of permutations of length $n$ avoiding all (respectively, containing exactly $q$ occurrences of) patterns from $\Pi$. Obviously, $\Pi(x)=\Pi(x, 0)$.

Remark 1.2. Hilbert series very similar to $\Pi(x)$ and $\Pi(x, t)$ are often considered in the theory of associative algebras. The well-known method of their study is based on the socalled bar-cobar duality which roughly means that a graded associative algebra $A$ and the $A_{\infty}$-coalgebra $\operatorname{Tor}_{.}^{A}(\mathbb{k}, \mathbb{k})$ are dual with respect to the functor Tor. As a corollary of this duality, one gets the fact that the Hilbert series of $A$ and of $\operatorname{Tor}_{.}^{A}(\mathbb{k}, \mathbb{k})$ are the inverses of each other, i.e., their product equals 1. (See [13] for the details on different computational methods for the Hilbert series of associative algebras and their homology.) It seems highly plausible that for an associative algebra with few relations, a combinatorial description of its homology is simpler than that of the algebra itself. However, for algebras with many relations, the situation is the opposite one.

Recall that the set of permutations avoiding an arbitrary fixed collection $\Pi$ has an important additional structure (see the appendix in [2]). Namely, in a suitable monoidal category it can be considered as the monomial basis of an algebra with monomial relations. (We refer the interested reader to the above appendix in [2] and references therein for the details. In particular, one can find the definition of the homology functor in the latter appendix.) Therefore, it seems natural to use the above mentioned homological duality in the theory of pattern avoidance. Combinatorial data appearing in this context is based on a generalization of the so-called cluster method of I. Goulden and D. Jackson, [6]. We explain below how one can get combinatorial information (for example, about the coefficients of the generating functions) of the corresponding graded homological vector spaces for collections of patterns with few entries.

To describe our results we need to recall the definition of a combinatorial gadget called clusters in [6]. They generalize the notion of a linkage given below.

A permutation $\sigma$ of length $n$ is called a linkage of an ordered pair of (not necessarily distinct) patterns $\left(\pi, \pi^{\prime}\right)$ of lengths $l$ and $l^{\prime}$ if (i) $n<l+l^{\prime}$; and (ii) the standardizations $\operatorname{st}[\sigma(1) \ldots \sigma(l)]$ and $\operatorname{st}\left[\sigma\left(n-l^{\prime}+1\right) \ldots \sigma(n)\right]$ are equal to $\pi$ and $\pi^{\prime}$ respectively. Since the length of $\sigma$ is less than the sum of the lengths of $\pi$ and $\pi^{\prime}$ one has that the standardizations of a right truncation of $\pi$ and a left truncation of $\pi^{\prime}$ are the same. Setting $k=\left(l+l^{\prime}-n\right)$, we say that a pair $\left(\pi, \pi^{\prime}\right)$ has a $k$-overlapping (or that $\left(\pi, \pi^{\prime}\right) k$-overlaps). In other words, a pair $\left(\pi, \pi^{\prime}\right) k$-overlaps whenever the standardization $s t[\pi(l-k+1) \ldots \pi(l)]$ is equal to the standardization $s t\left[\pi^{\prime}(1) \ldots \pi^{\prime}(k)\right]$. Notice that there can be several different linkages of two given patterns $\pi$ and $\pi^{\prime}$.

A cluster is a way to link together several patterns from a given set. More precisely, a $q$-cluster w.r.t. a given collection of patterns $\Pi$ is a triple $\left(\sigma ; \pi_{1}, \ldots, \pi_{q} ; d_{1}, \ldots, d_{q}\right)$ where $\sigma$ is a permutation, $\left\{\pi_{i}\right\}$ is a list of (not necessarily distinct) patterns from $\Pi$, and $\left\{d_{i}\right\}$ is a list of positive integers such that

(i) for every $j=1, \ldots, q$, st $\left[\sigma\left(d_{j}\right), \ldots, \sigma\left(d_{j}+l_{j}-1\right)\right]=\pi_{j} \in \Pi$, where $l_{j}$ is the length of $\pi_{j}$ (here $d_{j}$ labels the beginning of the pattern $\pi_{j}$ in $\sigma$ ); 
(ii) $d_{j+1}>d_{j}$ (patterns are listed from left to right) and $d_{j+1}<d_{j}+l_{j}$ (adjacent patterns are linked);

(iii) $d_{1}=1$, and the length of $\sigma$ is equal to $d_{q}+l_{q}-1$ (i.e., $\sigma$ is completely covered by the patterns $\left.\pi_{1}, \ldots, \pi_{q}\right)$.

Denote by $c l_{n, q}(\Pi)$ the number of $q$-clusters of length $n$ in a collection $\Pi$ and introduce the exponential generating function

$$
\Pi_{c l}(x, t)=x+\sum_{n>1, q \geqslant 1} c l_{n, q} \frac{x^{n}}{n !} t^{q} .
$$

(Here we use a natural convention that there always exists exactly one (fictitious) 0-cluster and, therefore, the above generating function starts with $x$.)

The following result is an immediate consequence of the general cluster method of I. Goulden and D. Jackson, [6] and its homological proof for the case of permutations can be found in $[2]$.

Theorem 1.3. In the above notation, one has:

$$
\Pi(x, t)=\frac{1}{1-\Pi_{c l}(x, t-1)} .
$$

Corollary 1.5. The exponential generating function $\Pi(x):=\Pi(x, 0)$ of the number of permutations avoiding the patterns from a given collection $\Pi$ satisfies the relation:

$$
\Pi(x)=\frac{1}{1-\Pi_{c l}(x,-1)} .
$$

Remark 1.7. In general, the problem of counting the number of $q$-clusters in a given collection of patterns $\Pi$ does not seem to be easier than counting the number of permutations of a given length avoiding $\Pi$. On the other hand, there exist natural classes of collections for which counting $q$-clusters is an easier task, see Section 3.

One can guess that since clusters can be described in terms of linkages of pairs of patterns the number of clusters can also be determined in terms of the combinatorics of possible intersections of these linkages. Exploiting the latter idea, we were able to prove the following theorem which is the main result of this paper.

Theorem 1.8. Two collections of patterns $\Pi_{1}$ and $\Pi_{2}$ are strongly Wilf equivalent if there exists a bijection $\varphi: \Pi_{1} \rightarrow \Pi_{2}$ respecting the following three properties:

- (lengths) For any $\pi \in \Pi_{1}$ its length equals to that of $\varphi(\pi) \in \Pi_{2}$;

- (linkages) A pair of patterns $\left(\pi, \pi^{\prime}\right)$ from $\Pi_{1}$ has a linkage of length $n$ if and only if the pair of its images $\left(\varphi(\pi), \varphi\left(\pi^{\prime}\right)\right)$ from $\Pi_{2}$ has a linkage of the same length $n$. 
- (overlapping sets) For each overlapping of any pair of patterns from $\Pi_{1}$ the bijection $\varphi$ preserves the subsets of entries that overlap. More precisely, for any pair $\left(\pi, \pi^{\prime}\right)$ of patterns $\pi, \pi^{\prime} \in \Pi_{1}$ of lengths $l$ and $l^{\prime}$ respectively and an arbitrary positive integer $k \leqslant \min \left(l, l^{\prime}\right)$, the coincidence of the standardizations $\operatorname{st}[(\pi(l-k+1) \ldots \pi(l))]=$ $\operatorname{st}\left[\left(\pi^{\prime}(1) \ldots \pi^{\prime}(k)\right)\right]$ implies the coincidence of the sets:

$\{\pi(l-k+1), \ldots, \pi(l)\}=\{\varphi(\pi)(l-k+1), \ldots, \varphi(\pi)(l)\}$, and $\left\{\pi^{\prime}(1), \ldots, \pi^{\prime}(k)\right\}=\left\{\varphi\left(\pi^{\prime}\right)(1) \ldots \varphi\left(\pi^{\prime}\right)(k)\right\}$.

The simplest case where Theorem 1.8 applies is to collections with a single pattern having no self-overlappings of length exceeding 1 . The following result implied by Theorem 1.8 was first conjectured by S. Elizalde in [3] and later proven in [2] by homological methods and, simultaneously, by J. Remmel whose methods were based on [10]. Namely,

Corollary 1.9. Two collections of patterns each containing a single permutation without nontrivial self-overlappings are strongly Wilf equivalent if

(i) the lengths of the permutations coincide;

(ii) the first entry and respectively the last entry of the permutations coincide.

A series of particular examples covered by Theorem 1.8 can be found in Section 5 of [1]. These examples are related to pairs of permutations having the separation property. We say that a pair of permutations $\alpha \in S_{k}$ and $\beta \in S_{k^{\prime}}$ has a separation property if $\beta$ avoids the pattern $\alpha(1) \ldots \alpha(k) k+1 \in S_{k+1}$ and $\alpha$ avoids $1 \beta(1)+1 \ldots \beta\left(k^{\prime}\right)+1 \in S_{k^{\prime}+1}$.

With each pair of permutations $\alpha \in S_{k}, \beta \in S_{k}^{\prime}$ and a natural number $l$ one can associate the subset $\Pi(\alpha, \beta ; l) \subset S_{k+l+k^{\prime}}$ of permutations defined by the following two properties. We say that $\pi \in \Pi(\alpha, \beta ; l)$ iff

(i) the standardizations of the $k$ first and $k^{\prime}$ last entries coincide with $\alpha$, and $\beta$ respectively;

(ii) the $k$ first entries are strictly smaller than the $k^{\prime}$ last entries; the $k^{\prime}$ last entries are strictly smaller than the remaining entries of $\pi$ in the middle. In other words, $\pi(i)<$ $\pi(j)<\pi(s)$ for any triple of indices $(i, j, s)$ such that $1 \leqslant i \leqslant k<s \leqslant k+l<j \leqslant$ $k+l+j$.

Corollary 1.10. Fix a pair of permutations $\alpha$ and $\beta$ having a separation property and $a$ $d$-tuple of natural numbers $\left(l_{1}, \ldots, l_{d}\right)$. Then all collections of $d$ distinct patterns $\left\{\pi_{1}, \ldots, \pi_{d}\right\}$ such that $\pi_{i} \in \Pi\left(\alpha, \beta ; l_{i}\right)$ are strongly Wilf equivalent.

Proof. The elements in the middle of each pattern never appear in the overlapping sets.

Let us present a few more examples illustrating how our theorem works in practice.

The following patterns

$$
1734526 \sim_{\mathrm{W}} 1735426 \sim_{\mathrm{W}} 1743526 \sim_{\mathrm{W}} 1745326 \sim_{\mathrm{W}} 1753426 \sim_{\mathrm{W}} 1754326
$$


are pairwise Wilf equivalent. They have self-overlappings of lengths 1 and 2 and coinciding pairs of the first two and the last two entries.

The following pair of Wilf equivalent patterns

$$
143265987 \sim_{\mathrm{W}} 134265897
$$

have self-overlappings of lengths 1 and 4, and the corresponding subsets of their initial and final entries of lengths 1 and 4 coincide while their initial and final subwords are different.

Finally, here is an example

$$
\{145623,13452\} \sim_{W}\{145623,13542\} \sim_{W}\{146523,13452\} \sim_{W}\{146523,13542\}
$$

of Wilf equivalent collections with 2 patterns in each.

In Section 2 we prove Theorem 1.8 and in Section 3 we apply our main construction to a class of collections of patterns and obtain a system of linear ordinary differential equations satisfied by $\Pi_{c l}(x, t)$ together with a set of similar generating functions defined below. In the follow-up [8] of the present paper we plan to study different asymptotic properties of $\Pi(x, t)$ using the suggested approach.

Acknowledgements. The authors are sincerely grateful to S. Kitaev for e-mail correspondence concerning this subject. We want to thank the anonymous referee for considerable efforts which allowed us to substantially improve the quality of the initial exposition.

\section{Proofs}

Our proof of Theorem 1.8 consists of an algorithm computing the cluster generating function $\Pi_{c l}(x, t)$ of a given collection of patterns $\Pi$. It will then be relatively easy to see that this algorithm uses only the lengths and the overlapping subwords for pairs of patterns from $\Pi$ considered as sets. To start with, we define for an arbitrary collection of patterns $\Pi$ a certain directed graph with labelled vertices and edges. The important fact is that the number of $q$ clusters with fixed initial and final subwords will be equal to the number of properly weighted paths of length $q$ in this graph with fixed initial and final vertices. The required weights can be computed using the edge labels. As a consequence, this graph uniquely determines the generating functions $\Pi_{c l}(x, t)$ and, therefore, $\Pi(x, t)$ (see Theorem 1.3).

Given an arbitrary collection of patterns $\Pi$ define its directed graph $\mathcal{G}(\Pi)$ with labelled vertices and edges as follows. The vertices of $\mathcal{G}(\Pi)$ will be labelled by permutations (of, in general, different lengths) and the labels of the edges are defined below.

- To define the vertices assume that some permutation $v$ is a left divisor of a pattern $\pi_{\alpha} \in \Pi$ and, at the same time, a right divisor of a (not necessarily different) pattern $\pi_{\beta} \in \Pi$. Then we assign to $v$ a vertex $v$ of $\mathcal{G}(\Pi)$ and, naturally, label this vertex by $v$. Notice that the same $v$ can arise from different pairs $\left(\pi_{\alpha}, \pi_{\beta}\right.$. In particular, the trivial 1-element permutation 1 comes from an arbitrary pair of not necessarily distinct patterns. (1) is called the distinguished vertex of $\mathcal{G}(\Pi)$ and the set of all vertices of $\mathcal{G}(\Pi)$ is denoted by $\mathcal{V}(\Pi) \ni(1)$.) 
- To define the edges take a pattern $\pi \in \Pi$ of some length $l$ and a pair $\left(\pi_{i}, \pi_{j}\right)$ of its initial and final subwords of lengths $k$ and $k^{\prime}$ (i.e., $\pi_{i}:=(\pi(1) \ldots \pi(k))$ and $\pi_{j}:=$ $\left.\left(\pi\left(l-k^{\prime}+1\right) \ldots \pi(l)\right)\right)$ such that standardizations $\operatorname{st}\left[\pi_{i}\right]$, st $\left[\pi_{j}\right]$ are the vertices of $\mathcal{G}(\Pi)$. Let $\mu_{i}$ and $\mu_{j}$ be the subsets of entries which appear in $\pi_{i}$ and $\pi_{j}$ respectively (i.e., $\mu_{i}:=\{\pi(1), \ldots, \pi(k)\}$ and $\left.\mu_{j}:=\left\{\pi\left(l-k^{\prime}+1\right), \ldots, \pi(l)\right\}\right)$. The triple $\left(\pi, \pi_{i}, \pi_{j}\right)$ then defines a directed edge from the vertex st $\left[\pi_{i}\right]$ to the vertex st $\left[\pi_{j}\right]$ which we label by the triple $\left(\mu_{i}, \mu_{j} ; l\right)$.

Remark 2.1. Notice that $\mu_{i}$ and $\mu_{j}$ are considered as unordered sets.

Notation. The vertices of $\mathcal{G}(\Pi)$ are labelled by permutations of different lengths. To distinguish the vertices from their underlying permutations we show them as encircled permutations, see e.g. Figure 1. Throughout the whole text, we will try to denote similar quantities by the same letter adding extra indices if required. For example, $l$ will typically mean the length of a pattern $\pi$ from a collection, $k$ will denote the length of a permutation $v$ which labels a vertex of $\mathcal{G}(\Pi)$ originating from a $k$-overlapping, $n$ will stand for the length of a cluster.

Four examples of $\mathcal{G}(\Pi)$ are given below. The upper left example is constructed from the collection $\Pi_{1}=\{1342765,152364\}$ of two patterns with no nontrivial overlappings. The upper right example comes from the single pattern $\{132679485\}$ having self-overlappings of lengths 1 and 3. The meaning of two other examples will be clear now.
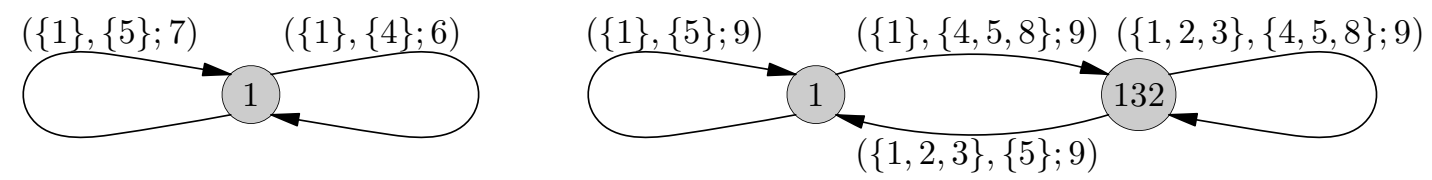

$\Pi_{1}=\{1342765,152364\}$

$$
\Pi_{2}=\{132679485\}
$$

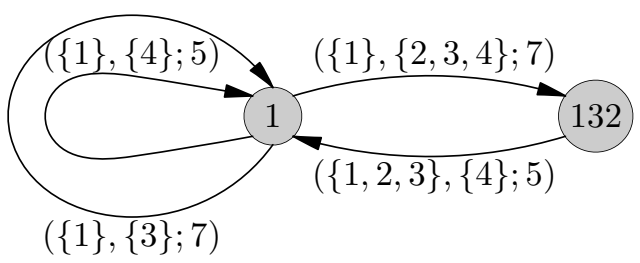

$$
\Pi_{3}=\{1576243,13254\}
$$

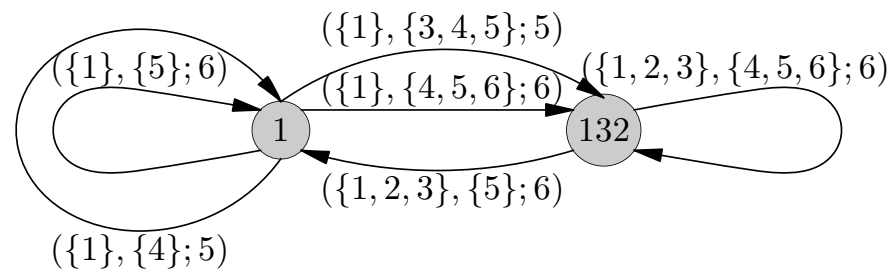

$$
\Pi_{4}=\{12354,132465\}
$$

Figure 1: Four examples of $\mathcal{G}(\Pi)$.

Our main technical result is as follows.

Theorem 2.2. The graph $\mathcal{G}(\Pi)$ uniquely determines the generating function $\Pi_{c l}(x, t)$.

The following corollary immediately implies Theorem 1.8. 
Corollary 2.3. Two collections of patterns $\Pi_{1}$ and $\Pi_{2}$ having isomorphic graphs $\mathcal{G}\left(\Pi_{1}\right)$ and $\mathcal{G}\left(\Pi_{2}\right)$ are strongly Wilf-equivalent. (Here by an "isomorphism" we mean a graph isomorphism preserving the labels of edges. The labels of vertices can change.)

Proof. To prove Theorem 2.2 we present a natural algorithm calculating the number of $q$ clusters in a given collection $\Pi$ using its graph $\mathcal{G}(\Pi)$. Namely, each vertex $(v)$ and a positive integer $n$ uniquely determine the subset $C l_{v, n, q}$ consisting of all $q$-clusters $\left(\sigma ; \pi_{1}, \ldots, \pi_{q}\right.$; $d_{1}, \ldots, d_{q}$ ), such that the length of $\sigma$ is equal to $n$ and the standardization of the initial subword of $\sigma$ is equal to $v$. Moreover, with each word $\bar{p}:=\left(p_{1} \ldots p_{k}\right)$ of length $k$ (where $k$ is the length of $v$ ) one can associate the subset $C l_{v, n, q}[\bar{p}] \subset C l_{v, n, q}$ consisting of those clusters in $C l_{v, n, q}$ which have $\bar{p}$ as their initial subword. We will explain how one can compute the cardinalities of $C l_{v, n, q}[\bar{p}]$ by induction on $q$ using the edge labels in $\mathcal{G}(\Pi)$. Therefore, the cardinalities of $C l_{v, n, q}$ can also be computed inductively as the sums over different $\bar{p}$. Since the standardization of any word of length 1 equals (1) the set $C l_{(1), n, q}$ coincides with the set of all $q$-clusters of length $n$. (The cardinality of the latter set is one of the coefficients in the cluster generating function $\Pi_{c l}(x, t)$.)

Let us now return to the induction step. Take an arbitrary vertex $(v) \in \mathcal{V}(\Pi)$ and let (v) $\stackrel{\pi_{1}}{\mapsto}\left(v_{1}, \ldots\right.$, , vo $\stackrel{\pi_{d}}{\mapsto}\left(v_{d}\right)$ be the list of all edges in $\mathcal{G}(\Pi)$ starting at the vertex $(v)$. Denote by $k_{j}$ the length of the permutation $v_{j}$ labeling the vertex $v_{j}$ and denote by $l_{j}$ the length of the pattern $\pi_{j}$. We present below a recurrence relation expressing the cardinality $c l_{v, n, q}[\bar{p}]$ of the set $C l_{v, n, q}[\bar{p}]$ in terms of the cardinalities $c l_{v_{j}, n-l_{j}+k_{j}, q-1}\left[\bar{p}^{\prime}\right]$ of $C l_{v_{j}, n-l_{j}+k_{j}, q-1}\left[\bar{p}^{\prime}\right]$ with the summation taken over a certain subset of words $\bar{p}^{\prime}$. Using this relation we can inductively calculate each $c l_{v, n, q}[\bar{p}]$ and then obtain the required $c l_{v, n, q}$ by summation over different $\bar{p}$. It will be convenient to subdivide the sets $C l_{v, n, q}$ and $C l_{v, n, q}[\bar{p}]$ into subsets indexed by the edges starting at the vertex (v). For example, $C l_{v \mapsto j_{j}, n, q}$ is the subset of $q$-clusters formed by linkages of length $n$ between the pattern $\pi_{j}$ and a $(q-1)$-cluster from $C l_{v_{j}, n-l_{j}+k_{j}, q-1}$. One has

$$
c l_{v, n, q}=\sum_{\substack{1 \leqslant p_{1}, \ldots, p_{k} \leqslant n, s t\left[\left(p_{1} \ldots p_{k}\right)\right]=v}} c l_{v, n, q}\left[p_{1} \ldots p_{k}\right]=\sum_{\substack{1 \leqslant p_{1}, \ldots, p_{k} \leqslant n, s t\left[\left(p_{1} \ldots p_{k}\right)\right]=v}} \sum_{j=1}^{d} c l_{\substack{\pi_{j} \\ v \mapsto v_{j}, n, q}}\left[p_{1} \ldots p_{k}\right] .
$$

Therefore, it is sufficient to find recurrence relations expressing the terms $c l_{v_{\pi_{j}} v_{j}, n, q}[\ldots]$ in the right-hand side of (2.4) using $c l_{v_{j}, n-l_{j}+k_{j}, q-1}[\ldots]$. To avoid very cumbersome notation let us take the case of a single edge starting at (v) which is equivalent to fixing $v_{j}$ in the above formulas. Let $v \stackrel{\pi}{\mapsto}(v)$ be an edge in a graph $\mathcal{G}(\Pi)$ coming from a pattern $\pi$ of length $l$ and let $k$ and $k^{\prime}$ be the lengths of the permutations labeling (v) and (v) respectively. To explain our recurrence we need to introduce the following extra notation associated to $\pi$.

Let $l>k+k^{\prime}$ and let $\psi \in S_{k+k^{\prime}}$ be the permutation which is the inverse of the standardization of the $k$ first and the $k^{\prime}$ last entries of $\pi$ and let $\bar{\psi}$ be the composition of $\psi$ with the shifting map $s h_{k, k^{\prime} \mapsto l}:\left\{1, \ldots, k, k+1, \ldots, k+k^{\prime}\right\} \rightarrow\{1, \ldots, k\} \cup\left\{l-k^{\prime}+1, \ldots, l\right\}$ defined by the formula:

$$
s h_{k, k^{\prime} \mapsto l}(j)=\left\{\begin{array}{l}
j, \text { if } j \leqslant k \\
j+l-k-k^{\prime}+1, \text { if } j>k .
\end{array}\right.
$$


In other words, $\bar{\psi}$ prescribes the rule how to write down the $k$ first and the $k^{\prime}$ last entries of the pattern $\pi$ in the increasing order:

$$
\left\{\pi(\bar{\psi}(1))<\pi(\bar{\psi}(2))<\ldots<\pi\left(\bar{\psi}\left(k+k^{\prime}\right)\right)\right\}=\{\pi(1), \ldots, \pi(k)\} \cup\left\{\pi\left(l-k^{\prime}+1\right), \ldots, \pi(l)\right\} .
$$

The following statement gives the required recurrence.

Lemma 2.5. The following relations hold:

- for $l>k+k^{\prime}$ set $\tilde{\pi}=\operatorname{st}\left[\pi(1) \ldots \pi(k) \pi\left(l-k^{\prime}+1\right) \ldots \pi(l)\right]$. Then

$$
\begin{aligned}
c l_{v \mapsto v^{\prime}, n, q} & {\left[p_{1} \ldots p_{k}\right]=\sum_{\begin{array}{c}
p_{k+1}, \ldots, p_{k+k^{\prime}} \\
s t\left[\left(p_{1} \ldots p_{k+k^{\prime}}\right)\right]=\tilde{\pi}
\end{array}}\left(\begin{array}{c}
p_{\psi(1)}-1 \\
\pi(\bar{\psi}(1))-1
\end{array}\right) \times } \\
& \times\left[\prod_{j=1}^{k+k^{\prime}-1}\left(\begin{array}{c}
p_{\psi(j+1)}-p_{\psi(j)}-1 \\
\pi(\bar{\psi}(j+1))-\pi(\bar{\psi}(j))-1
\end{array}\right)\right] \times\left(\begin{array}{c}
n-p_{\psi\left(k+k^{\prime}\right)} \\
l-\pi\left(\bar{\psi}\left(k+k^{\prime}\right)\right)
\end{array}\right) \times \\
& \times c l_{v^{\prime}, n-l+k^{\prime}, q-1}\left[p_{k+1}-\pi\left(l-k^{\prime}+1\right)+v^{\prime}(1), \ldots, p_{k+k^{\prime}}-\pi(l)+v^{\prime}\left(k^{\prime}\right)\right] .
\end{aligned}
$$

- for $l \leqslant k+k^{\prime}$ one has:

$$
\begin{aligned}
& c l_{v \mapsto v^{\prime}, n, q}\left[p_{1} \ldots p_{k}\right]= \\
& \quad=\sum_{\substack{p_{k+1}, \ldots, p_{l}: \\
\operatorname{st}\left[\left(p_{1} \ldots p_{l}\right)\right]=\pi}} c l_{v^{\prime}, n-l+k^{\prime}, q-1}\left[p_{l-k^{\prime}+1}-\pi\left(l-k^{\prime}+1\right)+v^{\prime}(1), \ldots, p_{l}-\pi(l)+v^{\prime}\left(k^{\prime}\right)\right] .
\end{aligned}
$$

Remark 2.8. The range of summation in (2.6) can be easily derived from our convention on the binomial coefficients claiming that $\left(\begin{array}{l}N \\ M\end{array}\right)=0$ if either $N<0$ or $M>N$. Moreover, we assume that $p_{j}$ 's are pairwise different positive integers not exceeding $n$. For the induction base we use the following initial data:

$$
C l_{v, n, 0}=\left\{\begin{array}{l}
\{1\}, \text { if } v=1 \text { and } n=1 \\
\varnothing, \text { otherwise. }
\end{array}\right.
$$

Proof. We show how to prove (2.6). In formula (2.6) one has the summation over all patterns $\sigma \in C l_{v \stackrel{\pi}{\leftrightarrow} v^{\prime}, n, q}$ such that the word $\left(\sigma(1) \ldots \sigma(k) \sigma\left(l-k^{\prime}+1\right) \ldots \sigma(l)\right)$ is fixed and coincides with $\left(p_{1} \ldots p_{k+k^{\prime}}\right)$. Indeed, the numbers $p_{j}$ are ordered by the permutation $\psi$ as follows: $p_{\psi(1)}<\ldots<p_{\psi\left(k+k^{\prime}\right)}$. Therefore, there are $\left(\begin{array}{c}p_{\psi(1)}-1 \\ \pi(\bar{\psi}(1))-1\end{array}\right)$ choices of entries less than $p_{\psi(1)}$ among the first $l$ entries of $\sigma$; there are $\left(\begin{array}{c}p_{\psi(2)}-p_{\psi(1)}-1 \\ \pi(\bar{\psi}(2))-\pi(\bar{\psi}(1))-1\end{array}\right)$ choices of entries greater than $p_{\psi(1)}$ and less than $p_{\psi(2)}, \ldots$; there are $\left(\begin{array}{c}n-p_{\psi\left(k+k^{\prime}\right)} \\ l-\pi\left(\bar{\psi}\left(k+k^{\prime}\right)\right)\end{array}\right)$ choices of entries greater than $p_{\psi\left(k+k^{\prime}\right)}$ among the first $l$ entries of $\sigma$; and $c l_{v^{\prime}, n-l+k^{\prime}, q-1}\left[p_{k+1}-\pi\left(l-k^{\prime}+1\right)+v^{\prime}(1), \ldots, p_{k+k^{\prime}}-\pi(l)+v^{\prime}\left(k^{\prime}\right)\right]$ ways to choose the remaining standardization of the last $\left(n-l+k^{\prime}\right)$ entries of $\sigma$.

In (2.7) the union of the $k$ initial entries and the $k^{\prime}$ final entries of $\pi$ covers the whole list of entries of $\pi$, i.e., the set $\{1, \ldots, l\}$. Therefore, all binomial coefficients appearing in (2.6) are equal to 1 which leads to $(2.7)$. 
As an immediate consequence of Lemma 2.5 one can see that the numbers $c l_{v \stackrel{\pi}{\mapsto} v^{\prime}, n, q}[\ldots]$ of $(q+1)$-clusters depend only on the length, the $k$ first and the $k^{\prime}$ last entries of $\pi$ considered as sets. This justifies the information we use as the edge labels of the graph $\mathcal{G}(\Pi)$. The formulas expressing $c l_{v, n, q}[\ldots]$ in terms of $c l_{\bullet, \bullet, q-1}[\ldots]$ depend only on the labeling of the edges starting at $(v)$. Therefore, these cardinalities can be computed by induction on $q$ using the edge labels of the graph $\mathcal{G}(\Pi)$. Finally, as we mentioned before, the set of all $q$-clusters of length $n$ of the whole collection $\Pi$ is equal to the set $C l_{(1), n, q}$.

\subsection{The case of a single pattern}

Let us consider separately the situation when $\Pi$ contains just a single pattern, since in this case some simplifications of our construction can be done.

First of all the following observation explains why the graph $\mathcal{G}(\{\pi\})$ is not required.

Lemma 2.9. Let $\pi$ be a pattern of length $l$ and let $\left(2 l-k_{1}\right), \ldots,\left(2 l-k_{d}\right)$ be the list of all distinct lengths of possible self-linkages of $\pi$, i.e., $k_{1}, \ldots, k_{d}$ is the list of distinct lengths of self-overlappings of $\pi$. Then $\mathcal{G}(\{\pi\})$ is a complete directed graph on d vertices with loops and with lengths of the underlying permutations being equal to $k_{1}, \ldots, k_{d}$. Each ordered pair of (not necessary distinct) vertices of $\mathcal{G}(\{\pi\})$ are connected by exactly one directed edge labeled by the corresponding initial and final subwords of $\pi$.

It is obvious that $k_{1}=1$ and denote by $k\left(k=k_{d}\right)$ the length of the largest overlapping. Let $v_{s}$ be the standardization of the $k_{s}$ first entries of $\pi$ (i.e., $v_{s}$ is the labeling permutation of the $s$-th vertex in $\mathcal{G}(\{\pi\}))$. Since all patterns involved in any cluster coincide with $\pi$, the standardization of the initial subword of any cluster is always the same. Hence for different $v_{s}$ and fixed $n$ and $q$ all the sets $C l_{v_{s}, n, q}$ coincide. Therefore, it makes sense to denote by $c l_{n, q}$ and $c l_{n, q}\left[p_{1} \ldots p_{k}\right]$ the cardinalities of the set of $q$-clusters of length $n$ and those having $\left(p_{1} \ldots p_{k}\right)$ as their initial subword respectively. We introduce the same set of notations for the self-overlappings of $\pi$ similar to what we have used in Lemma 2.5 for the case $k_{s}<l-k$.

Namely, for $l>k+k_{s}$ let $\psi \in S_{k+k_{s}}$ be the permutation which is the inverse of the standardization of the $k$ first and the $k_{s}$ last entries of $\pi$; for $l \leqslant k+k_{s}$ let $\psi$ be the inverse of $\pi$. Let $\overline{\psi_{s}}$ be the composition $s h_{k, k_{s} \mapsto l} \circ \psi_{s}$ using which one gets the following rearrangement of the first $k$ and last $k_{s}$ elements of $\pi$ in increasing order:

$\left\{\pi\left(\overline{\psi_{s}}(1)\right)<\pi\left(\overline{\psi_{s}}(2)\right)<\ldots<\pi\left(\overline{\psi_{s}}\left(k+k_{s}\right)\right)\right\}=\{\pi(1), \ldots, \pi(k)\} \cup\left\{\pi\left(l-k_{s}+1\right), \ldots, \pi(l)\right\}$.

Additionally, let $\tilde{\pi}_{s}$ be the standardization of the $k$ first and $k_{s}$ last entries of $\pi$.

In the case of a single pattern Lemma 2.5 implies the following result.

Lemma 2.10. For a single pattern the recurrence formula for the numbers of $q$-clusters is 
as follows:

$$
\begin{aligned}
& c l_{n, q}\left[p_{1} \ldots p_{k}\right]=\sum_{\substack{s: \\
k_{s}<l-k}} \sum_{\begin{array}{c}
1 \leqslant p_{k+1}, \ldots, p_{2 k} \leqslant n, \\
s t\left[\left(p_{1} \ldots p_{k+k_{s}}\right)\right]=\tilde{\pi}_{s}
\end{array}}\left(\begin{array}{c}
p_{\psi_{s}(1)}-1 \\
\pi\left(\overline{\psi_{s}}(1)\right)-1
\end{array}\right) \times \\
& \times {\left[\prod_{j=1}^{k+k_{s}-1}\left(\begin{array}{c}
p_{\psi_{s}(j+1)}-p_{\psi_{s}(j)}-1 \\
\pi\left(\overline{\psi_{s}}(j+1)\right)-\pi\left(\overline{\psi_{s}}(j)\right)-1
\end{array}\right)\right] \times\left(\begin{array}{c}
n-p_{\psi_{s}\left(k+k_{s}\right)} \\
l-\pi\left(\overline{\psi_{s}}\left(k+k_{s}\right)\right)
\end{array}\right) \times } \\
& \times c l_{n-l+k_{s}, q-1}\left[p_{k+1}-\pi\left(l-k_{s}+1\right)+v_{s}(1), \ldots, p_{s}\left(k+k_{s}\right)-\pi(l)+v_{s}\left(k_{s}\right), p_{k+k_{s}+1}, \ldots, p_{2 k}\right]+ \\
&+\sum_{\substack{s: \\
k_{s} \geqslant l-k}}^{\substack{1 \leqslant p_{k+1}, \ldots, p_{l+k-k_{s}} \leqslant n, s t\left[\left(p_{1} \ldots p_{l}\right)\right]=\pi}} \quad(2.11)
\end{aligned}
$$

(As above we assume that the set of 0-clusters contains the unique fictitious element of length 1 while the set of 1-clusters contains the single pattern $\pi$.)

\section{Application}

In this section we discuss a specific class of collections of patterns. Our method from Section 2 allows us to construct a system of linear ordinary differential equations in variable $x$ for the cluster generating functions $\Pi_{c l}(x, t)$ together with a set of similar generating functions associated to a given collection of patterns $\Pi$. The main definition is as follows.

Definition 3.1. A collection of patterns $\Pi$ is called monotone if for all $k>0$ and for each pair of (not necessarily distinct) patterns $\left(\pi, \pi^{\prime}\right)$ from $\Pi$ the existence of their $k$-overlapping implies that the initial subword of the pattern $\pi^{\prime}$ does not contain entries greater than $k$.

The following lemma explains how the monotonicity assumption simplifies the structure of the set of clusters and their generating functions.

Lemma 3.2. Let $\sigma$ be a linkage of a pair of patterns $\left(\pi, \pi^{\prime}\right)$. Suppose that the initial subword of $\pi$ of length $k$ does not contain entries greater than $k$ and that the initial subword of $\pi^{\prime}$ of length $k^{\prime}$ does not contain entries greater than $k^{\prime}$ (where $k^{\prime}$ is the length of the overlapping of the pair $\left(\pi, \pi^{\prime}\right)$ in $\left.\sigma\right)$. Then the initial subword of length $k$ of $\sigma$ is equal to the initial subword of $\pi$, i.e., $\sigma(j)=\pi(j)$ for $1 \leqslant j \leqslant k$.

Proof. Let $l$ and $l^{\prime}$ be the lengths of $\pi$ and $\pi^{\prime}$ respectively. Then the length of $\sigma$ equals to $n=l+l^{\prime}-k^{\prime}$. Set $j=\pi^{-1}(1)$ and $j^{\prime}=\pi^{\prime-1}(1)$ respectively. The number $j$ is the minimal entry of $\pi$ and therefore is less than or equal to $k\left(j^{\prime} \leqslant k^{\prime}\right.$ respectively). Therefore, $\sigma(j)$ is the smallest entry among the $l$ first entries of the linkage $\sigma$ and $\sigma\left(n-l^{\prime}+j^{\prime}\right)$ is the smallest entry among the $l^{\prime}$ last entries of $\sigma$. In particular, $\sigma(j)<\sigma\left(n-l^{\prime}+j^{\prime}\right)$. This implies that $\sigma(j)$ is the smallest entry in the whole of $\sigma$ and hence $\sigma(j)=\pi(j)=1$. Similar arguments based on simple properties of standardizations imply that $\sigma\left(\pi^{-1}(2)\right)$ is the only entry in $\sigma$ greater than 1 , etc. 
Lemma 3.2 implies that for an arbitrary vertex $v \in \mathcal{V}(\Pi)$ all clusters in the subset $C l_{v, n, q}$ always have $v$ as their initial subword. This means that the number $c l_{v, n, q}\left[p_{1} \ldots p_{k}\right]$ is non-vanishing if and only if $\left[p_{1} \ldots p_{k}\right]=[v(1) \ldots v(k)]$, where $k$ is the length of $v$. Consider two vertices $v$ and $v$ of lengths $k$ and $k^{\prime}$ connected by an edge $v \stackrel{\pi}{\mapsto} \vartheta$ in $\mathcal{G}(\Pi)$. We can simplify formula (2.6) using our assumption on the intersections of clusters. At first consider the case when the length $l$ of the pattern $\pi$ is greater than $k+k^{\prime}$. One observes that in this case, there is no summation in (2.6) since the summands in the left-hand and the right-hand sides of (2.6) are non-vanishing if and only if for $1 \leqslant j \leqslant k$ one has that $p_{j}=v(j)=\pi(j)$ and for $1 \leqslant j \leqslant k^{\prime}$ one has that $p_{k+j}=\pi\left(l-k^{\prime}+j\right)$. Therefore, only the last binomial coefficient in the product in the right-hand side of (2.6) is different from 1. Denote by $m$ the maximal entry of the final subword of $\pi$. (In notation of Lemma 2.5 we have $m=\pi\left(\bar{\psi}\left(k+k^{\prime}\right)\right)$.) Since we do not have to specify the arguments of the functions $c l_{v, n, q}[\ldots]$, the resulting recurrence relation for the cardinalities of the set of clusters is:

$$
c l_{v \stackrel{\pi}{\mapsto} v^{\prime}, n, q}=\left(\begin{array}{c}
n-m \\
l-m
\end{array}\right) c l_{v^{\prime}, n-l+k^{\prime}, q-1} .
$$

The case $l \leqslant k+k^{\prime}$ is also covered by (3.3) since in this case the maximal entry $m$ equals to the length $l$ of the permutation $\pi$ and the corresponding binomial coefficient is equal to 1 .

Introduce the following family of generating functions:

$$
y_{v}(x, t):=\sum_{n, q} c l_{v, n, q} \frac{x^{n}}{n !} t^{q},
$$

one for each vertex $v$ of $\mathcal{G}(\Pi)$. Let $v \stackrel{\pi_{1}}{\mapsto} v_{1}, \ldots, v \stackrel{\pi_{d}}{\mapsto} \vartheta_{d}$ be the list of all edges in $\mathcal{V}(\Pi)$ starting at $v$. Denote by $l_{j}$ the length of the pattern $\pi_{j}$; denote by $k_{j}$ the length of $v_{j}$, and, finally, denote by $m_{j}$ the maximal entry among the $k_{j}$ last entries of $\pi_{j}$. Formula (3.3) implies the following reccurence relation:

$$
c l_{v, n, q}=\sum_{j=1}^{d}\left(\begin{array}{c}
n-m_{j} \\
l-m_{j}
\end{array}\right) c l_{v_{j}, n-l_{j}+k_{j}, q-1} ; \quad c l_{v, n, 0}=\left\{\begin{array}{l}
1, v=1 \\
0, v \neq 1 .
\end{array}\right.
$$

Theorem 3.5. Given a monotone collection of patterns $\Pi$ one has that the cluster generating function $\Pi_{c l}(x, t)=y_{1}(x, t)$ together with all $y_{v}(x, t), v \in \mathcal{V}(\Pi)$ solve the following system of linear ordinary differential equations in $x$ :

$$
\frac{d^{m}}{d x^{m}} y_{v}(x, t)=t \sum_{j=1}^{d} \frac{d^{m-m_{j}}}{d x^{m-m_{j}}}\left(\frac{x^{l_{j}-m_{j}}}{\left(l_{j}-m_{j}\right) !} \frac{d^{k_{j}}}{d x^{k_{j}}} y_{v_{j}}(x, t)\right) .
$$

Here $m:=\max \left\{m_{j}\right\}$ and $(v)$ runs over the set $\mathcal{V}(\Pi)$ of all vertices of $\mathcal{G}(\Pi)$. (Boundary conditions for each $y_{v}(x, t)$ can be easily determined in each particular case using the initial terms in (3.4).)

Proof. Follows from (3.4). 
Remark 3.7. As an immediate consequence of Theorem 3.5 one gets that $\Pi_{c l}(x, t)=y_{1}(x, t)$ satisfies a certain high order linear ordinary differential equation with polynomial coefficients (which can be obtained from the above system after the elimination of all $y_{v}(x, t),(v \neq(1)$.)

In particular, one can get the following simplification of Theorem 1.8 for monotone collections of patterns.

Corollary 3.8. Two monotone collections of patterns $\Pi_{1}$ and $\Pi_{2}$ are strongly Wilf equivalent if there exists a bijection $\varphi: \Pi_{1} \rightarrow \Pi_{2}$ preserving the first two properties as in Theorem 1.8 (i.e., preserving lengths and linkages) and, additionally, preserving the maxima of the overlapping sets. More precisely, for any pair $\left(\pi, \pi^{\prime}\right)$ of patterns $\pi, \pi^{\prime} \in \Pi_{1}$ of lengths $l$ and $l^{\prime}$ respectively and an arbitrary positive integer $k \leqslant \min \left(l, l^{\prime}\right)$ the coincidence of the standardizations $\operatorname{st}[(\pi(l-k+1) \ldots \pi(l))]=\operatorname{st}\left[\left(\pi^{\prime}(1) \ldots \pi^{\prime}(k)\right)\right]$ implies the coincidence of the maxima of the sets:

$$
\max \{\pi(l-k+1), \ldots, \pi(l)\}=\max \{\varphi(\pi)(l-k+1), \ldots, \varphi(\pi)(l)\} .
$$

(Two other sets $\left\{\pi^{\prime}(1), \ldots, \pi^{\prime}(k)\right\}$ and $\left\{\varphi\left(\pi^{\prime}\right)(1) \ldots \varphi\left(\pi^{\prime}\right)(k)\right\}$ coincide with the standard set $\{1, \ldots, k\}$ because of the monotonicity property.)

With this in mind one can add two more strongly Wilf equivalent patterns to the pair (1.11) discussed in the introduction. Namely,

$$
143265987 \sim_{\mathrm{W}} 134265897 \sim_{\mathrm{W}} 143256987 \sim_{\mathrm{W}} 134256897 .
$$

\subsection{Case of a single monotone pattern}

Let us present further simplifications for monotone collections with a single pattern. As it was shown in Section 2.1 we do not really need the graph $\mathcal{G}(\Pi)$ for collections with one pattern. Take a monotone pattern $\pi$ of length $n$ and let $1=k_{1}<\ldots<k_{d}=k$ be the list of all different lengths of self-overlappings of $\pi$. Let $\pi(l)=m_{1} \leqslant \ldots \leqslant m_{d}=m$ be the list of maxima among the corresponding number of the last entries of $\pi$, i.e., $m_{j}:=\max \left\{\pi\left(l-k_{j}+1\right), \ldots, \pi(l)\right\}$.

Corollary 3.9. Given a monotone pattern $\pi$, one has the following recurrence formula for the set of its q-clusters of length $n$ :

$$
c l_{n, q}=\sum_{j=1}^{d}\left(\begin{array}{c}
n-m_{j} \\
l-m_{j}
\end{array}\right) c l_{n-l_{j}+k_{j}, q-1} .
$$

Further, its cluster generating function $y_{1}(x, t)=\Pi_{c l,\{\pi\}}(x, t)$ solves the linear ordinary differential equation:

$$
\frac{d^{m}}{d x^{m}} y_{1}(x, t)=t \sum_{j=1}^{d} \frac{d^{m-m_{j}}}{d x^{m-m_{j}}}\left(\frac{x^{l-m_{j}}}{\left(l-m_{j}\right) !} \frac{d^{k_{j}}}{d x^{k_{j}}} y_{1}(x, t)\right),
$$

with the boundary conditions: $y_{1}(0, t)=0, y_{1}^{\prime}(0, t)=1, y_{1}^{\prime \prime}(0, t)=\ldots=y_{1}^{(m-1)}(0, t)=0$. 
Two particular cases covered by this corollary, namely the pattern $(12 \ldots l)$ and an arbitrary pattern of the form $(1 \ldots a)$ having no nontrivial self-overlappings were considered earlier in [5].

Finally, using these considerations we can completely describe which permutations of length $5\left(\pi \in S_{5}\right)$ are Wilf equivalent. Our main Theorem 1.8 gives necessary and sufficient condition for Wilf equivalence of two patterns of length 5 . Notice that there are two natural transformations of patterns preserving the cluster generating functions. The first one is the reversion that rewrites a pattern backwards, i.e., it sends $(\pi(1), \ldots, \pi(n))$ to $(\pi(n), \ldots, \pi(1))$. The second one takes the complement of a pattern, i.e., it sends $(\pi(1), \ldots, \pi(n))$ to $(n-$ $\pi(1)+1, \ldots, n-\pi(n)+1)$. They generate the group $\mathbb{Z}_{2} \times \mathbb{Z}_{2}$ acting on $S_{5}$. One can easily check that this action has 32 orbits of which 4 orbits with representatives 12345, 14325, 21354, 25314 have length 2 and the remaining 28 have length 4 . Additionally, there are 14 orbits whose permutations have no nontrivial selp-overlappings; 15 orbits with the only nontrivial selfoverlapping of length 2; 2 orbits with the only nontrivial self-overlapping of length 3, and a single orbit with self-overlappings of length 2, 3 and 4 (see the lists of representatives in Proposition 3.11).

Proposition 3.11. Subdividing the representatives of the $\mathbb{Z}_{2} \times \mathbb{Z}_{2}$-orbits on $S_{5}$ into subsets according to the lengths of their maximal possible self-overlappings, one gets the following:

- Wilf equivalent orbits having no nontrivial overlappings are enumerated by their first and last elements:

$$
\begin{gathered}
13452 \sim_{W} 13542 \sim_{W} 14352 \sim_{W} 14532 \sim_{W} 15342 \sim_{W} 15432 \\
12453 \sim_{W} 12543 ; \quad 12354 \sim_{W} 13254 ; \quad 21354 \sim_{W} 21534 ; \quad 24153 \sim_{W} 25143 .
\end{gathered}
$$

- among all 15 orbits with only 2-overlappings having the representatives

$12435,12534,13425,13524,14325,14523,15324,15423,15234$,

$21453,21543,23514,24513,25314,25413$

no two are strongly Wilf equivalent to each other;

- 2 orbits with representatives 14253, 15243 having 3-overlappings are not Wilf equivalent;

- the unique orbit with overlappings of lengths 2,3 and 4 is represented by the monotone pattern 12345.

Proof. Besides the application of Theorem 1.8 one has to check by hand that the number of 3 -clusters is distinguishing all the 15 orbits with only 2-overlappings and that the number of 2-clusters already distinguishes between the two orbits having only 3-overlappings. 


\subsection{Examples of differential equations}

Let us finish the paper by presenting the (system of) linear differential equations for our examples in Fig.1. One can easily check that all the collections $\Pi_{1}-\Pi_{4}$ shown there are monotone. Set $\pi_{1}=1342765$ and $\pi_{2}=152364$ for $\Pi_{1}$ and notice that its graph contains a single vertex. Then, in the above notation, one has $l_{1}=7, m=m_{1}=5, l_{2}=6, m_{2}=$ $4, k=1$. Equation (3.6) for the cluster generating function $y_{1}(x, t)$ then takes the form:

$$
y_{1}^{\mathrm{V}}=t\left(\frac{x^{2}}{2} y_{1}^{\prime}+\frac{d}{d x}\left(\frac{x^{2}}{2} y_{1}^{\prime}\right)\right)
$$

For the collection $\Pi_{2}=\{132679485\}$ that has self-overlappings of lengths 1 and 3 one has $l_{1}=9, k_{1}=1, k=k_{2}=3, m_{1}=5, m=m_{2}=8$. With this data, equation (3.10) takes the form:

$$
y_{1}^{\mathrm{VIII}}=t\left(\frac{d^{3}}{d x^{3}}\left(\frac{x^{3}}{3 !} y_{1}^{\prime}\right)+x y_{1}^{\prime \prime \prime}\right) .
$$

For the collection $\Pi_{3}=\{1576243,13254\}$, denote its edges by $A=(\{1\},\{3\}, 7), B=$ $(\{1\},\{4\}, 5), C=(\{1\},\{2,3,4\}, 7)$ and $D=(\{1,2,3\},\{4\}, 5)$. Then, one has $l_{A}=7, k_{A}=$ $1, m_{A}=3 ; l_{B}=5, k_{B}=1, m_{B}=4 ; l_{C}=7, k_{C}=3, m_{C}=4 ; l_{D}=5, k_{D}=1, m_{D}=4$. Thus, one gets the following system of equations:

$$
\left\{\begin{array}{l}
y_{1}^{\mathrm{IV}}=t\left(\frac{d}{d x}\left(\frac{x^{4}}{4 !} y_{1}^{\prime}\right)+x y_{1}^{\prime}+\frac{x^{3}}{3 !} y_{132}^{\prime \prime \prime}\right) \\
y_{132}^{\mathrm{IV}}=t x y_{1}^{\prime}
\end{array}\right.
$$

In this case it is easy to get a linear ordinary differential equation satisfied by $y_{1}$ by dividing both sides of the first equation by $\frac{x^{3}}{3 !}$, differentiating the result with respect to $x$ and equating the expressions for $y_{132}^{\mathrm{IV}}$ from the first and second equations. The resulting equation has the form

$$
6\left(x^{3} y_{1}^{\mathrm{V}}-3 x^{2} y_{1}^{\mathrm{IV}}-t x^{3}\left(\frac{x^{4}}{4 !} y_{1}^{\prime}\right)^{\prime \prime}+3 t x^{2}\left(\frac{x^{4}}{4 !} y_{1}^{\prime}\right)^{\prime}-t x^{3}\left(x y_{1}^{\prime}\right)^{\prime}+3 t x^{3} y_{1}^{\prime}\right)-t x^{7} y_{1}^{\prime}=0
$$

Finally, for the collection $\Pi_{4}=\{12534,132465\}$, denote its edges by $A=(\{1\},\{4\}, 5)$; $B=(\{1\},\{5\}, 6) ; C=(\{1\},\{3,4,5\}, 5) ; D=(\{1\},\{4,5,6\}, 6) ; E=(\{1,2,3\},\{5\}, 6)$, and $F=(\{1,2,3\},\{4,5,6\}, 6)$. Then, one has $l_{A}=5, k_{A}=1, m_{A}=4 ; l_{B}=6, k_{B}=1, m_{B}=5$; $l_{C}=5, k_{C}=3, m_{C}=5 ; l_{D}=6, k_{D}=3, m_{D}=6 ; l_{E}=6, k_{E}=1, m_{E}=5 ; l_{F}=6, k_{F}=$ $3, m_{F}=6$. Thus, one gets the following system of equations:

$$
\left\{\begin{array}{l}
y_{1}^{\mathrm{VI}}=t\left(\frac{d^{2}}{d x^{2}}\left(x y_{1}^{\prime}\right)+\frac{d}{d x}\left(x y_{1}^{\prime}\right)+y_{132}^{\mathrm{IV}}+y_{132}^{\prime \prime \prime}\right) \\
y_{132}^{\mathrm{VI}}=t\left(\frac{d}{d x}\left(x y_{1}^{\prime}\right)+y_{132}^{\prime \prime \prime}\right)
\end{array}\right.
$$

Let us extract from this system a linear ordinary differential equation satisfied by $y_{1}$. To simplify our notation set $u=y_{1}^{\prime}, v=y_{132}^{\prime \prime \prime}$. Then we get the system:

$$
\left\{\begin{array}{l}
u^{\mathrm{V}}=t\left((x u)^{\prime \prime}+(x u)^{\prime}+v^{\prime}+v\right) \\
v^{\prime \prime \prime}=t\left((x u)^{\prime}+v\right)
\end{array}\right.
$$

THE ELECTRONic JOURNAL OF COMBINATORICS 18(2) (2011), \#P9 
Since the coefficients in the left-hand sides are both equal to 1 , we can eliminate $v$ by differentiating the first equation a number of times and substituting $v^{\prime \prime \prime}$ from the second equation till we can solve both equations for the remaining $v$ and its derivative of appropriate order. In our concrete case, differentiating the first equation twice and substituting $v^{\prime \prime \prime}$ from the second equation, we get

$$
\left\{\begin{array}{l}
u^{\mathrm{VII}}=t\left((x u)^{\mathrm{IV}}+(x u)^{\prime \prime \prime}+t(x u)^{\prime}+t v+v^{\prime \prime}\right) \\
v^{\prime \prime \prime}=t\left((x u)^{\prime}+v\right)
\end{array}\right.
$$

Differentiating the first equation in the latter system once and substituting $v^{\prime \prime \prime}$ again, we get

$$
\left\{\begin{array}{l}
u^{\mathrm{VIII}}=t\left((x u)^{\mathrm{V}}+(x u)^{\mathrm{IV}}+t(x u)^{\prime \prime}+t(x u)^{\prime}+t\left(v^{\prime}+v\right)\right), \\
v^{\prime \prime \prime}=t\left((x u)^{\prime}+v\right) .
\end{array}\right.
$$

Finally, equating the expressions for $v+v^{\prime}$ from the first equation in the latter system and from the original equation for $u^{\mathrm{V}}$ we get the following equation

$$
u^{\mathrm{VIII}}-t(x u)^{\mathrm{V}}-t u^{\mathrm{V}}-t(x u)^{\mathrm{IV}}+t^{2}(x u)^{\prime \prime \prime}-t^{2}(x u)^{\prime \prime}+t^{3}(x u)^{\prime}-t^{2}(x u)^{\prime}=0
$$

containing $u$ and its derivatives only. Substituting $u=y_{1}^{\prime}$ we obtain the required equation

$$
y_{1}^{\mathrm{IX}}-t\left(x y_{1}^{\prime}\right)^{\mathrm{V}}-t y_{1}^{\mathrm{VI}}-t\left(x y_{1}^{\prime}\right)^{\mathrm{IV}}+t^{2}\left(x y_{1}^{\prime}\right)^{\prime \prime \prime}-t^{2}\left(x y_{1}^{\prime}\right)^{\prime \prime}+t^{3}\left(x y_{1}^{\prime}\right)^{\prime}-t^{2}\left(x y_{1}^{\prime}\right)^{\prime}=0
$$

for the cluster generating function.

Remark 3.12. Notice that since the leading terms in the left-hand sides of system (3.6) are always equal to 1 elimination process similar to the one just described will always lead to an equation satisfied by the cluster generating function $\Pi(x, t)$. On the other hand, there is no guarantee that the obtained linear ordinary equation with polynomial coefficients will have the minimal possible order among such equations satisfied by $\Pi(x, t)$.

\section{References}

[1] R. E. L. Aldred, M. D. Atkinson, and D. J. McCaughan, Avoiding consecutive patterns in permutations. Advances in Applied Mathematics, 45 (2010), no. 3, 449-461.

[2] V. Dotsenko, A. Khoroshkin, Anick-type resolutions and consecutive pattern avoidance. arXiv:1002.2761.

[3] S. Elizalde Torrent, Consecutive patterns and statistics on restricted permutations. Ph.D. thesis, Universitat Politècnica de Catalunya, (2004), 170 pp.

[4] R. Ehrenborg, S. Kitaev, and P. Perry, A spectral approach to consecutive patternavoiding permutations. arXiv:1009.2119.

[5] S. Elizalde, M. Noy, Consecutive patterns in permutations. Formal power series and algebraic combinatorics (Scottsdale, AZ, 2001). Adv. in Appl. Math. 30 (2003), no. 1-2, 110-125. 
[6] I. P. Goulden, D. M. Jackson, An inversion theorem for cluster decompositions of sequences with distinguished subsequences. J. London Math. Soc. (2) 20 (1979), no. 3, $567-576$.

[7] S. Kitaev, A survey on partially ordered patterns. In Permutation Patterns (2010), S. Linton, N. Ruskuc, and V. Vatter, Eds., vol. 376 of London Mathematical Society Lecture Note Series, Cambridge University Press, pp. 115-135.

[8] A. Khoroshkin, B. Shapiro, Asymptotic results in consecutive pattern avoidance of permutations, in preparation.

[9] J. Liese, J. Remmel, Generating functions for permutations avoiding a consecutive pattern. Ann. Comb., 14 (2010) 123-141.

[10] A. Mendes, J. Remmel, Permutations and words counted by consecutive patterns. Adv. in Appl. Math. 37 (2006), no. 4, 443-480.

[11] J. Noonan, D. Zeilberger, The Goulden-Jackson cluster method: extensions, applications and implementations. J. Differ. Equations Appl. 5 (1999), no. 4-5, 355-377.

[12] D. Rawlings, The q-exponential generating function for permutations by consecutive patterns and inversions. JCTA 114 (2007) 184-193.

[13] V. A. Ufnarovskij, Combinatorial and asymptotic methods in algebra. Algebra, VI, 1-196, Encyclopaedia Math. Sci., 57, Springer, Berlin, 1995.

[14] H. S. Wilf, The patterns of permutations. Kleitman and combinatorics: a celebration (Cambridge, MA, 1999). Discrete Math. 257 (2002), no. 2-3, 575-583. 